\title{
Estudo da sinterização de nanopartículas por microscopia eletrônica de transmissão in situ
}

\section{(In situ transmission electron microscopy study of nanoparticle sintering)}

\author{
M. A. L. Cordeiro ${ }^{1}$, E. R. Leite ${ }^{2}$ \\ ${ }^{1}$ Departamento de Engenharia de Materiais, ${ }^{2}$ Departamento de Química \\ Universidade Federal de S. Carlos, Rod. Washington Luiz, km 235, C. P. 676, S. Carlos, SP, Brasil 13565-905
}

\begin{abstract}
Resumo
O controle e o entendimento do processo de sinterização é de grande destaque científico e tecnológico devido à inerente dependência entre as características de um material e sua micro/nanoestrutura. Entretanto, o crescente controle na produção de nanopartículas trouxe novos desafios à sinterização, especialmente na aplicação dos modelos clássicos de transporte de massa. Diante desses desafios, técnicas in situ de caracterização têm surgido para a investigação de processos físicos e químicos durante sua ocorrência, em especial a sinterização. Dentre essas técnicas, a microscopia eletrônica de transmissão in situ mostra-se um importante método na investigação do processo de sinterização pela visualização direta dos fenômenos envolvidos. Nesse trabalho é exposto a potencialidade da técnica pelo estudo da sinterização de nanopartículas de $\mathrm{CeO}_{2}$ por microscopia eletrônica de transmissão in situ com a análise dos fenômenos de densificação, orientação cristalográfica e ligantes nas superfícies dessas partículas.

Palavras-chave: sinterização, MET in situ, dióxido de cério, nanopartículas.
\end{abstract}

Abstract

The control and the understanding of the sintering process are of utmost scientific and technological importance due to the intrinsic dependence of the characteristic of a a material on its micro/nanostructure. The increasing control of the production of nanoparticles led to new challenges to sintering, especially on the application of classical mass transport models. Taking this into account, in situ characterization techniques have been proposed to investigate physical and chemical processes that occur during sintering. Among those techniques, the in situ transmission electron microscopy (TEM) is an important characterization technique to investigating sintering enabling a direct observation of the phenomena occurring during sintering. In this work the potentiality to the study of $\mathrm{CeO}$, nanoparticles by in situ TEM is reported, consisting on the analysis of the densification phenomenon, the crystallographic orientation and the binding species at the nanoparticle surface.

Keywords: sintering, in situ TEM, cerium dioxide, nanoparticles.

\section{INTRODUÇÃO}

A sinterização é um primordial processo tecnológico com ampla utilização na produção de materiais cerâmicos e na metalurgia do pó. Devido à intrínseca relação entre propriedades de um material policristalino e sua micro/ nanoestrutura, como tamanho e forma dos grãos, porosidade, distribuição de poros e natureza e distribuição de fases secundárias, o controle e o entendimento do processo de sinterização é de grande importância científica e tecnológica [1]. Geralmente a sinterização é definida como o tratamento térmico para a consolidação de pós ou materiais porosos, previamente conformados, em uma peça específica. Os estudos mais importantes de seus mecanismos começaram na década de 50 do século passado empregando técnicas ex situ de investigação de materiais policristalinos [2]. Essa metodologia é baseada na retirada do material em sinterização em um determinado instante (após um gradiente de resfriamento) e sua microestrutura estudada por várias técnicas de microscopia. Desses estudos foram obtidas importantes informações sobre a variação de tamanho de grão, morfologia dos poros, coalescência das partículas, etc. Em geral esses trabalhos foram concentrados em materiais policristalinos ou particulados com tamanhos de grãos superiores a $0,2 \mu \mathrm{m}$. Apesar das limitações, devido à complexidade real de um sistema de pós compactados, as teorias resultantes desses experimentos auxiliaram no entendimento da evolução microestrutural de muitos materiais produzidos [1-3].

Entretanto, nas últimas duas décadas o controle eficiente na síntese de nanopartículas (partículas de tamanhos entre 1 e $100 \mathrm{~nm}$ ) de diversos materiais e formatos trouxe novos desafios à sinterização, especialmente com o uso de pós nanoparticulados [4]. Apesar dos importantes avanços no entendimento do processo de sinterização já alcançados, a compreensão dos reais fenômenos de transferência de massa entre nanoparticulas durante o processo de sinterização ainda não é bem compreendido. Vários questionamentos ainda são relevantes, como por exemplo, se o processo de densificação de nanopartículas segue modelos teóricos clássicos (e.g., modelo escalar de Herring [5, 6]), se há outros mecanismos de transporte de massa além daqueles que a teoria prevê, 
que fração volumétrica das nanopartículas corresponde o transporte por difusão superficial (especialmente em partículas menores que $5 \mathrm{~nm}$ ), entre outras indagações.

Além disso, a natureza ex situ dos experimentos limitaram as medições apenas para a verificação da morfologia e tamanhos das partículas e pescoços após ciclo(s) de queima dos materiais. Esse método tem a desvantagem de não poder identificar os processos de transferência de massa durante o processo de sinterização, sendo examinado somente o resultado final da sinterização e não a dinâmica do processo. Em contraste a essa abordagem, novas metodologias e técnicas experimentais que possuem a capacidade de análise do processo durante sua ocorrência conquistaram uma grande importância, abrindo um caminho para revelar a dinâmica de eventos complexos pela análise direta de fenômenos físicos e químicos.

Umas das técnicas mais promissoras é a microscopia eletrônica de transmissão (MET) in situ [7, 8], na qual se permite acompanhar os mais diversos processos físicos e químicos pela observação direta dos fenômenos em resolução atômica. MET in situ data da década de 1960 quando importantes problemas em Ciência dos Materiais, como a fadiga de metais para a aplicação em aviação e a influência da radiação em materiais tinham de ser entendidos [9-11]. Nesses estudos era possível aplicar certas tensões mecânicas nas amostras durante as análises por MET, ou mesmo estudar a influência da radiação por elétrons. Mas apenas na década de 90 a observação direta da sinterização foi conseguida com resolução suficiente para distinguir planos cristalinos durante o experimento $[12,13]$ e somente na última década se mostrou uma ferramenta primordial para o estudo transporte de massa entre partículas [14], juntamente à evolução dos equipamentos de MET e de porta-amostras especiais.

Desse modo, esse estudo tem como objetivo prover uma visão geral das possiblidades que a técnica de MET in situ pode conceder para o entendimento da sinterização de nanopartículas pela visualização direta dos fenômenos envolvidos durante o processo em si.

\section{MATERIAIS E MÉTODOS}

Para o estudo da sinterização por MET in situ foram utilizados nanocristais de $\mathrm{CeO}_{2}$ sintetizados pela rota hidrolítica por duas fases, em autoclave de aço inoxidável [15]. Em uma síntese típica, foram adicionados na autoclave, de capacidade $50 \mathrm{~mL}, 15 \mathrm{~mL}$ de solução aquosa de $17 \mathrm{mmol} / \mathrm{L}$ de nitrato de cério (III) $\left(\mathrm{Ce}\left(\mathrm{NO}_{3}\right)_{3}\right), 15 \mathrm{~mL}$ de tolueno, 0,5 $\mathrm{mL}$ de ácido oleico (OLA, surfactante) e $0,15 \mathrm{~mL}$ de tertbutilamina. Após fechado o sistema, este foi submetido a $180{ }^{\circ} \mathrm{C}$ com taxa de aquecimento $10^{\circ} \mathrm{C} /$ min por $24 \mathrm{~h}$, sem agitação. Decorrido esse tempo, deixou-se o sistema esfriar até a temperatura ambiente naturalmente. A parte superior da mistura (parte orgânica) foi separada e centrifugada para a retirada de impurezas. Alíquotas de etanol foram adicionadas à solução resultante até a precipitação dos nanocristais de $\mathrm{CeO}_{2}$. Após mais quatro ciclos de lavagens das partículas (precipitação por adição de etanol, separação dos precipitados e redispersão em clorofórmio) obteve-se soluções coloidais dos nanocristais de $\mathrm{CeO}_{2}$ em clorofórmio.

Os experimentos por MET in situ foram realizados em um microscópio eletrônico de transmissão FEI Tecnai F20 com canhão de elétrons por emissão de campo operando a 200 kV. Organizações de nanocristais de $\mathrm{CeO}_{2}$ sobre grades de níquel com filme amorfo de carbono foram preparadas por simples gotejamento do coloide. $\mathrm{O}$ aquecimento das amostras no microscópio eletrônico de transmissão foi realizado utilizando um porta-amostra com estágio a quente de inconel (Gatan 628), sendo os experimentos realizados sob vácuo $\left(\sim 10^{-5} \mathrm{~Pa}\right)$. Séries de experimentos preliminares foram realizadas para se determinar a temperatura em que os fenômenos em questão sucediam em um período de tempo conveniente.

\section{RESULTADOS E DISCUSSÃO}

As Figs. 1a e $1 \mathrm{~b}$ mostram as micrografias dos nanocristais de $\mathrm{CeO}_{2}$ sintetizados pela rota hidrolítica por duas fases. As partículas apresentaram um comportamento de autoorganização em duas dimensões (2D) no substrato, com coordenação de partículas igual a 6 (prioritariamente). As distâncias entre partículas $(\sim 2,2 \mathrm{~nm})$ são equivalentes ao tamanho dos grupos oleatos ligados quimicamente à superfície das partículas (provenientes da síntese) [15] e inferem que a organização é mediada pelo surfactante. Adicionalmente, tanto as distâncias interplanares (indicadas na micrografia da Fig. 1 b) e o difratograma de raios $\mathrm{X}$ do pó dos nanocristais de $\mathrm{CeO}_{2}$ evidenciaram a cristalinidade dos materiais produzidos. O histograma da Fig. 1c mostra tamanho médio dos nanocristais de $6,7 \mathrm{~nm}$, com baixa dispersão de tamanhos.

A Fig. 2 mostra micrografias resolvidas no tempo de um típico experimento de sinterização de nanocristais $\mathrm{CeO}_{2}$ a $870{ }^{\circ} \mathrm{C}$ por $10 \mathrm{~min}$. É importante ressaltar que o início da aquisição das imagens ocorreu após a estabilização térmica de todo o sistema, pois as vibrações ocasionadas pelas dilatações do substrato impediram qualquer aquisição útil de informação imediatamente no início do patamar de temperatura. A estabilização, em todos os experimentos, sempre ocorreu entre 3 e 5 min depois de atingido o patamar de temperatura desejado. Portanto, o ponto de partida de qualquer fenômeno observado tem início de tempo $(\mathrm{t}=0 \mathrm{~s})$ após a estabilização do sistema.

A primeira característica observada é a estabilidade dos nanocristais. A maior parte dos nanocristais permaneceu sem mudanças significativas até $840{ }^{\circ} \mathrm{C}$. Acima de $840{ }^{\circ} \mathrm{C}$ os nanocristais começaram a se movimentar e se aproximar, sendo que alguns nanocristais $(<10 \%)$ já apresentavam a formação de pescoços entre partículas. Devido à mobilidade, nesta fase os nanocristais se apresentaram em domínios (ou grupos) em contraste à quase homogeneidade de ordenação em temperatura ambiente (Figs. 1a-1b). Porém, somente a $870{ }^{\circ} \mathrm{C}$ a formação das estruturas de pescoços foi mais frequente $(>75 \%)$ concomitantemente ao processo de sinterização. 

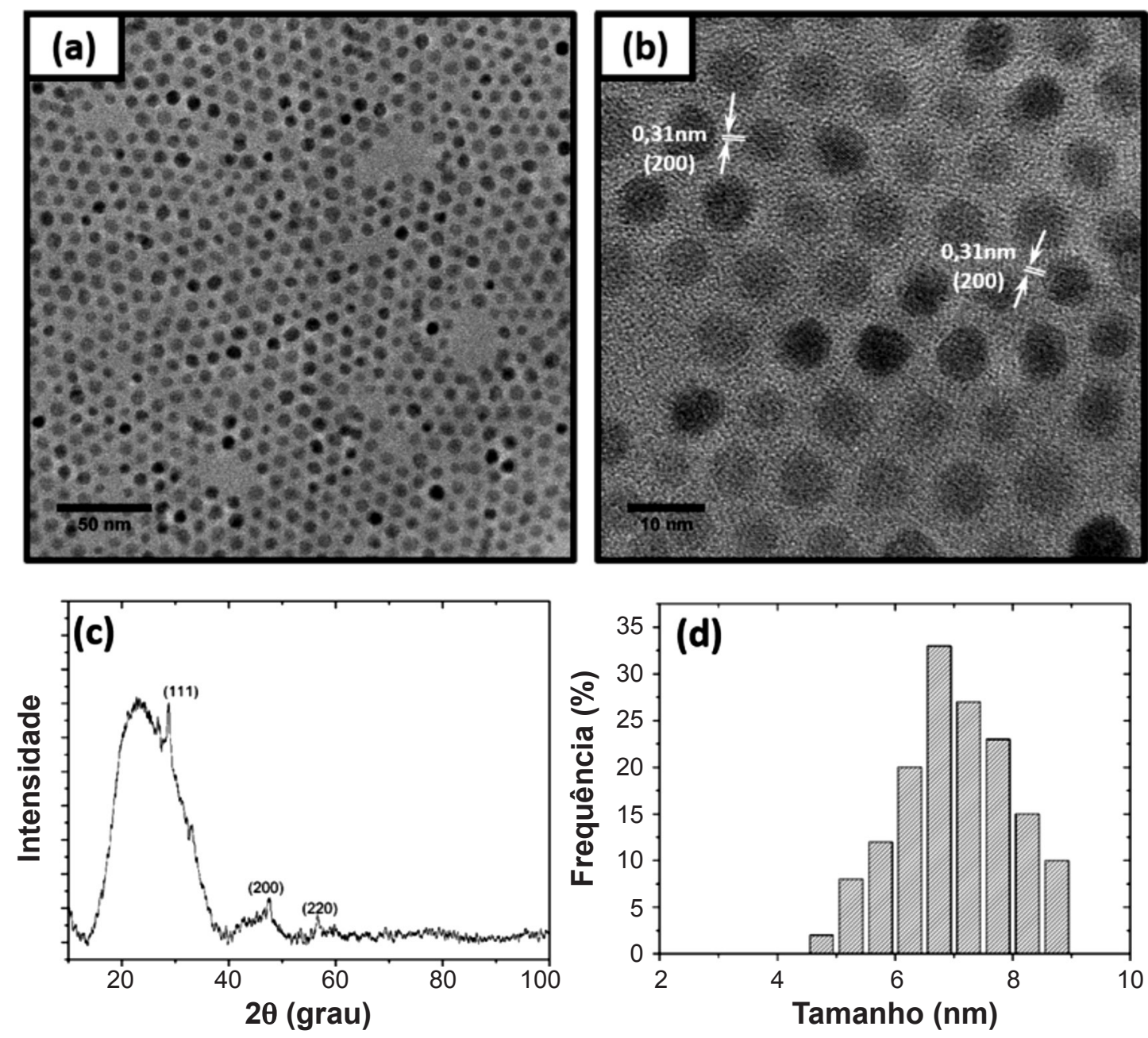

Figura 1: (a) e (b) Micrografias obtidas em microscópio eletrônico de transmissão, (c) DRX e (d) histograma de dispersão de tamanhos dos nanocristais de $\mathrm{CeO}_{2}$.

[Figure 1: (a) and (b) TEM micrographs, (c) XRD pattern and (d) histogram of the dispersion of CeO nanocrystal size.]

Uma possível explicação para a alta estabilidade térmica dos nanocristais de $\mathrm{CeO}_{2}$ até $840{ }^{\circ} \mathrm{C}$ seria devido aos grupo orgânicos (grupos oleatos provenientes da síntese) ligados quimicamente à superfície das partículas. Quando os nanocristais são aquecidos em ambiente inerte (vácuo), o material orgânico da superfície passa por um processo de combustão incompleta, que geralmente ocorre aproximadamente na faixa $400-500{ }^{\circ} \mathrm{C}$, formando uma capa de carbono amorfo ao redor do nanocristal. Tal camada pode ser a responsável pela estabilidade de forma dos nanocristais em temperaturas maiores que $500{ }^{\circ} \mathrm{C}$. Quando a temperatura é alta o suficiente, a camada amorfa pode se desorganizar e então os nanocristais podem se movimentar ao longo do substrato e entrar em contato para a formação do pescoço entre as partículas. Concomitante à saída da camada de carbono dos nanocristais, as possíveis facetações de alguns cristais passam a se suavizar, tornando o cristal esférico propriamente. Esse fenômeno reforça a hipótese que a camada de carbono amorfo garante a estabilidade das partículas que, quando ausente proporciona a reorganização de forma do nanocristal para atingir um estado de forma de menor energia (esférico). Por outro lado, a presença de uma camada orgânica/amorfa sobre os nanocristais pode evitar que processo de sinterização ocorra pelo bloqueio estérico ao transporte de massa entre partículas. Em geral, a presença de impurezas na superfície das nanopartículas dificulta o transporte de massa entre partículas pela formação de uma barreira ao processo, em oposição a casos onde tais impurezas promovem a densificação [4] (e.g., como na sinterização ativada [16]).

Adicionalmente, os estudos sobre a sinterização de pós nanométricos de $\mathrm{CeO}_{2}$ mostraram uma dependência bimodal do comportamento de retração de amostras compactadas [17-19]. Nesses trabalhos foram identificadas duas faixas de temperaturas onde houve maior retração de amostras de pós nanométricos compactados $\left(750-800{ }^{\circ} \mathrm{C}\right.$ e acima de $\left.1600{ }^{\circ} \mathrm{C}\right)$ [17], enquanto para amostras compactadas de pós micrométricos foi observada apenas uma faixa de maior retração (acima de $1600{ }^{\circ} \mathrm{C}$ ) [18], i.e., a faixa de maior sinterabilidade $700-800{ }^{\circ} \mathrm{C}$ seria exclusiva para os pós nanométricos. Em ambos sistemas as faixas de temperatura foram identificadas como regiões de perda de oxigênio (redução) do $\mathrm{CeO}_{2}[17,20]$. Esse processo foi apontado como o promotor do processo de sinterização dos materiais 

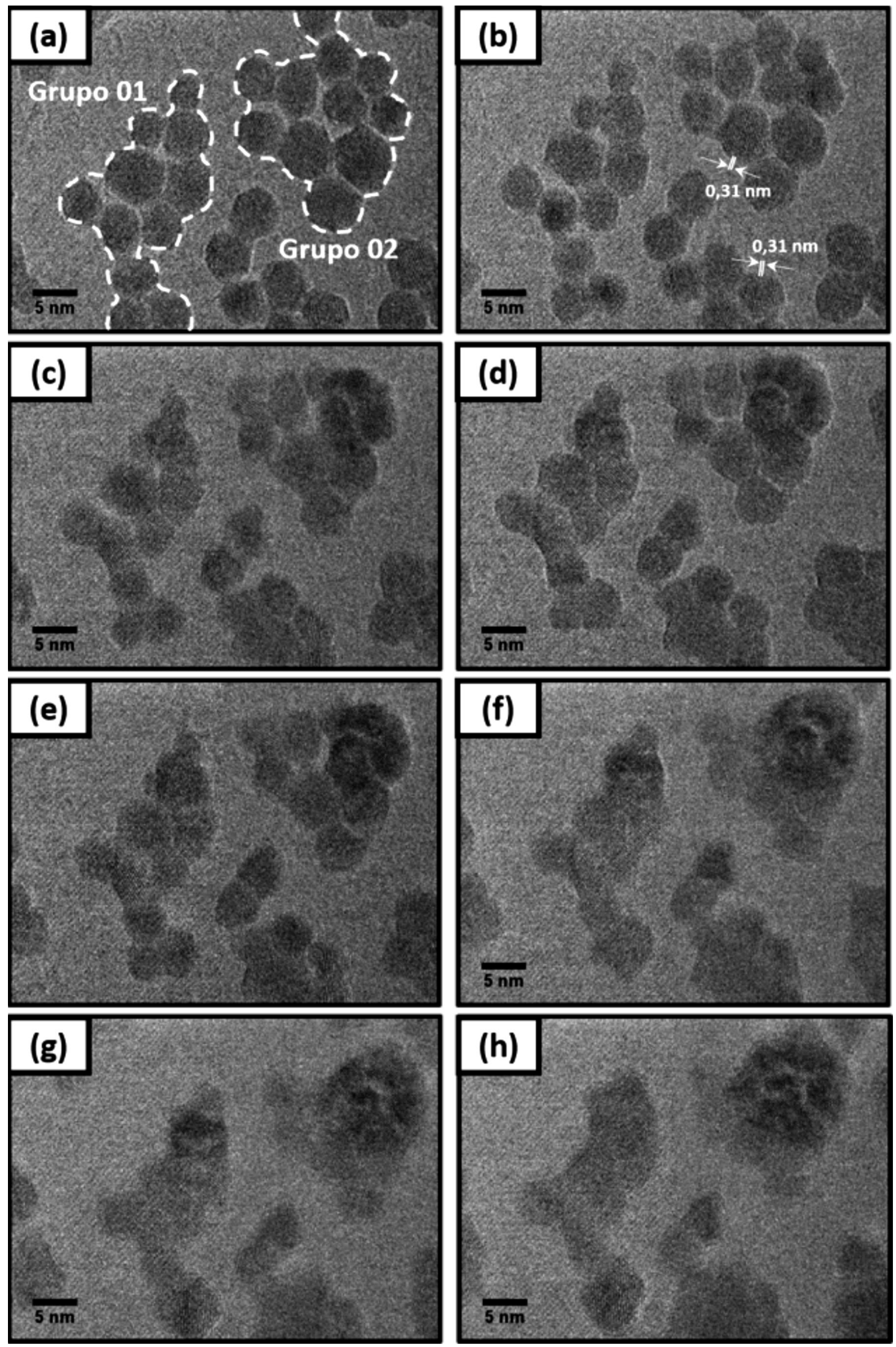

Figura 2: Sequência de micrografias obtidas em microscópio eletrônico de transmissão, resolvidas no tempo da sinterização de nanocristais de $\mathrm{CeO}_{2}$ a $870{ }^{\circ} \mathrm{C}$. (a) $20 \mathrm{~s}$; (b) $61 \mathrm{~s}$; (c) $203 \mathrm{~s}$; (d) $312 \mathrm{~s}$; (e) $380 \mathrm{~s}$; (f) $422 \mathrm{~s}$; (g) $501 \mathrm{~s}$; (h) $596 \mathrm{~s}$.

[Figure 2: Sequence of TEM images obtained during the experiment with $\mathrm{CeO}_{2}$ nanocrystals at $870{ }^{\circ} \mathrm{C}$. (a) $20 \mathrm{~s}$; (b) $61 \mathrm{~s}$; (c) $203 \mathrm{~s}$; (d) $312 \mathrm{~s}$; (e) $380 \mathrm{~s}$; (f) $422 \mathrm{~s}$; (g) $501 \mathrm{~s}$; (h) $596 \mathrm{~s}$. 


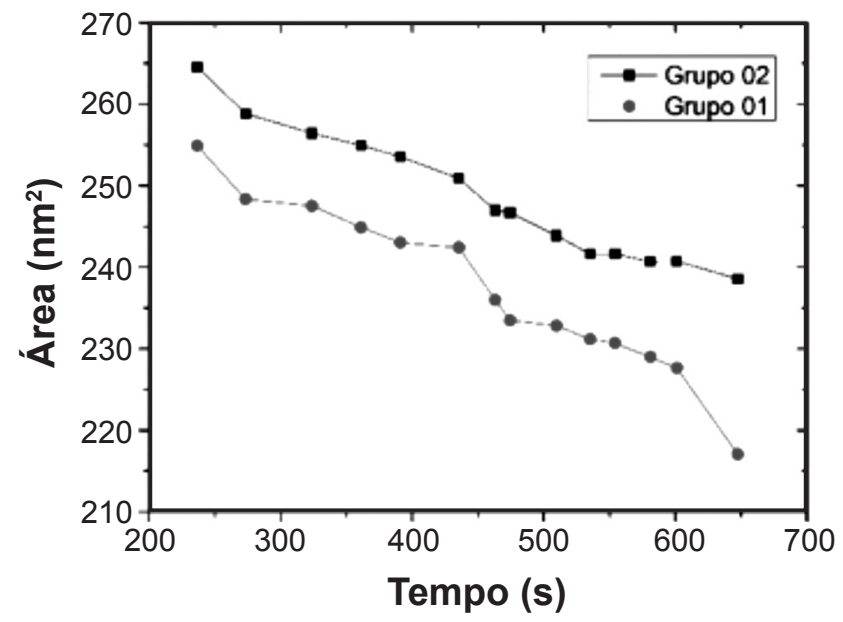

Figura 3: Valores das áreas dos grupos de nanopartículas de $\mathrm{CeO}_{2}$ em função do tempo de experimento.

[Figure 3: Values of the areas of the CeO2 nanoparticles as a function of the time of the experiment.]
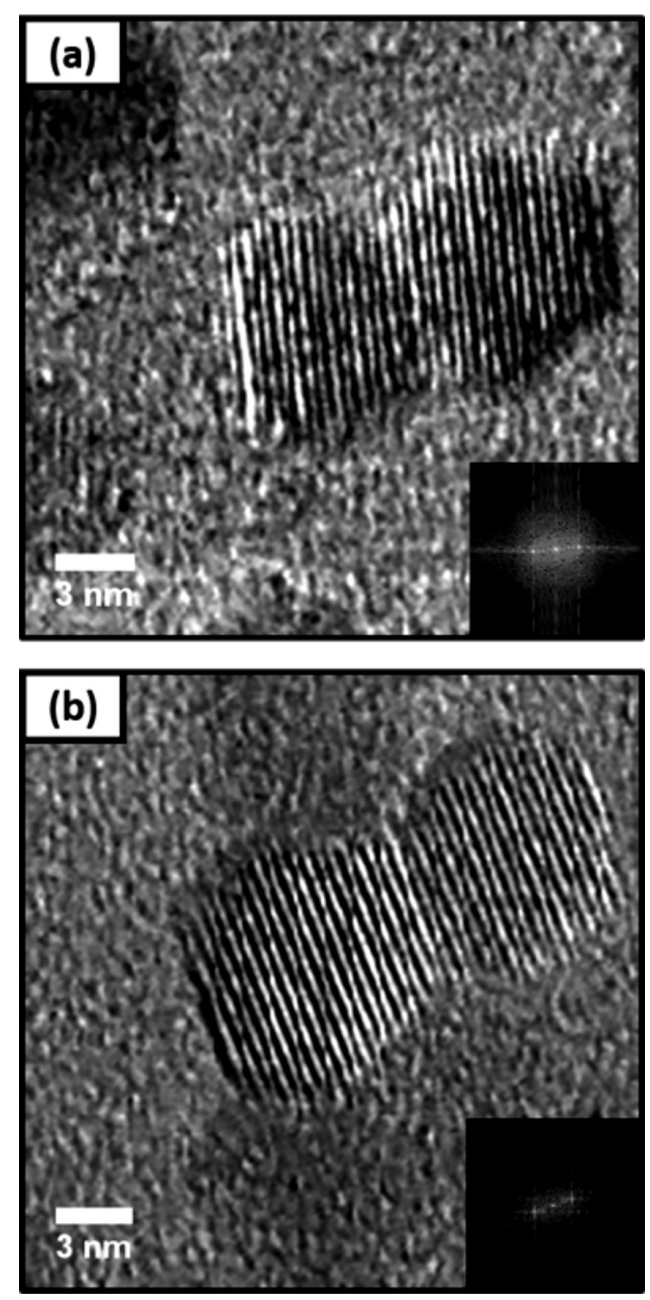

Figura 4: (a) e (b) Micrografias obtidas em microscópio eletrônico de transmissão de dois grupos de nanopartículas de $\mathrm{CeO}_{2}$ à $870{ }^{\circ} \mathrm{C}$ mostrando coerência entre orientações cristalográficas, juntamente às respectivas FFT.

[Figure 4: (a) e (b) TEM micrographs of two $\mathrm{CeO}_{2}$ nanoparticles at $870{ }^{\circ} \mathrm{C}$ showing coherence in the crystallographic orientations, and the corresponding FFT.] pela formação de vacâncias de oxigênio e consequente maior mobilidade dos ânions. Em acordo com esses trabalhos, a melhor temperatura de sinterização determinada empiricamente no presente estudo foi próxima à primeira temperatura de maior retração de amostras compactadas de pós nanométricos $\left(870^{\circ} \mathrm{C}\right)$.

Para a análise do processo de sinterização das partículas nesse experimento, foram agrupados dois sistemas de nanocristais (pontilhados na Fig. 3a) em processo de sinterização ao longo do tempo. Em todo curso do experimento foi possível observar que os nanocristais em questão permaneceram cristalinos com evidente visualização dos parâmetros de rede referentes ao $\mathrm{CeO}_{2}$ (indicações na Fig. 2b). Inicialmente os nanocristais sofreram progressivas aproximações ao longo do experimento com a conseguinte
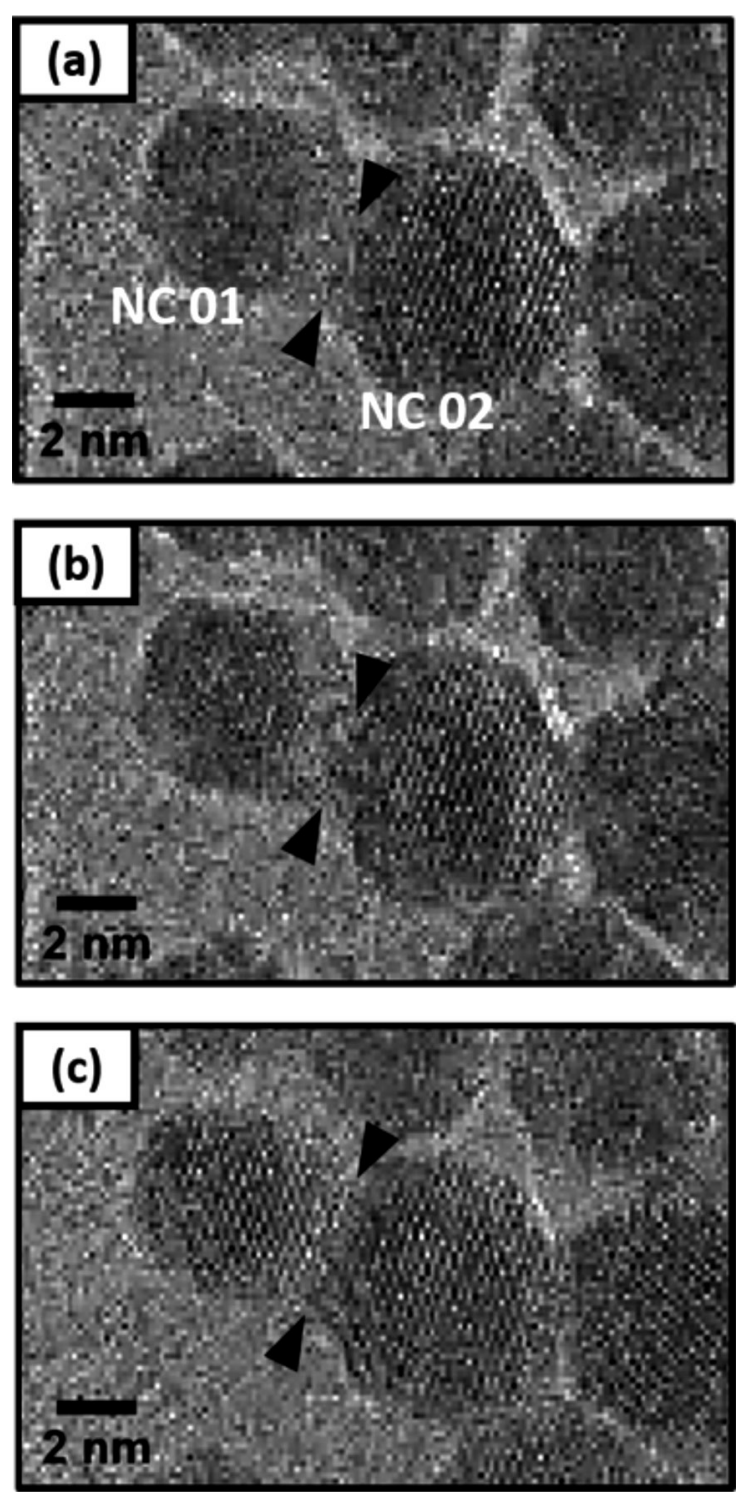

Figura 5: (a) e (b) Micrografias obtidas em microscópio eletrônico de transmissão de duas nanopartículas de $\mathrm{CeO}_{2}$ a $870{ }^{\circ} \mathrm{C}$ ao longo do experimento. (a) $15 \mathrm{~s}$; (b) $66 \mathrm{~s}$; (c) $82 \mathrm{~s}$.

[Figure 5: (a) e (b) TEM micrographs of two $\mathrm{CeO}_{2}$ nanoparticles at $870^{\circ} \mathrm{C}$ during the experiment. (a) $15 \mathrm{~s}$; (b) $66 \mathrm{~s}$; (c) $82 \mathrm{~s}$.] 
formação de pescoços. Esse comportamento de aproximação entre nanocristais e aumento de tamanho e área dos pescoços durante o processo de sinterização indica que o processo tem característica densificante. De fato, o acompanhamento das áreas dos dois grupos de nanocristais (Fig. 3) evidenciase que houve a retração de ambos os sistemas ao longo do tempo, sendo que suas velocidades de retração são próximas, demostrando mecanismos equivalentes de densificação.

Nas imagens da Fig. 2 observa-se que alguns nanocristais já iniciam o experimento com a formação de pescoço entre as partículas, com a sinterização já em ocorrência. A principal causa desse comportamento é a combinação entre planos cristalinos coerentes logo quando esses nanocristais entram em contato. A Fig. 4 mostra alguns desses nanocristais justamente com a transformada de Fourier, onde se pode verificar a coerência/alinhamento entre os planos cristalinos. Embora a possibilidade de transferência de massa entre nanocristais em sinterização possa ocorrer sem a completa coerência entre planos de partículas em alguns casos [14], o alinhamento cristalográfico promove a melhor condição energética para a promoção de transferência de massa [14, 21]. Em todos os casos de sinterização logo no início dos experimentos, a coerência entre orientações cristalográficas foram prioritárias, o que facilitou a formação do pescoço entre partículas e a promoção da sinterização.

Por outro lado, para a ocorrência da sinterização de alguns nanocristais houve um processo de rearranjo dos nanocristas, de modo que a combinação coerente dos respectivos planos cristalinos pudessem ocorrer. A Fig. 5 mostra duas partículas do mesmo experimento em sinterização e suas transformadas de Fourier. Inicialmente, os dois nanocristais estavam em contato, porém não possuíam orientações cristalográficas coerentes sem a progressão da sinterização nesse estágio (Fig. 5a). Com o passar do tempo de experimento, um dos nanocristais começou a se reorientar (Fig. 5b) e passou a ter a mesma orientação cristalográfica de seu par (Fig. 5c) e, então, o processo de sinterização prossegue. Embora os dois nanocristais estivessem em contato durante todo o tempo, a formação do pescoço entre as partículas só pode ser efetivamente formada após a reorientação da partícula $\mathrm{NC}$ 01, como indicado na Fig. 5c.

Adicionalmente, as reorientações de nanocristais observadas para o progresso da sinterização ocorreram nos nanocristais de menor coordenação de partículas. No caso das partículas da Fig. 5, o nanocristal (NC 01) de coordenação 3 se reorienta em relação ao seu par de coordenação 5 , o que pode ser explicado pelo maior grau de liberdade das partículas de menor coordenação para ao rearranjo. Embora a força motriz para esse processo de reorientação ainda seja discutida em vários estudos, simulações mostram que a diminuição da energia livre de superfície seja a mais provável, juntamente à valências não satisfeitas ("dangling bonds") dos átomos da superfície das partículas [22, 23].

\section{CONCLUSÕES}

A técnica de microscopia eletrônica de transmissão in situ mostra-se um importante método na investigação de processos físicos e químicos durante sua ocorrência, principalmente no estudo da sinterização de nanopartículas. Esse trabalho expôs a densificação de nanopartículas de $\mathrm{CeO}_{2}$ durante a sinterização por MET in situ, com notório acompanhamento dos planos cristalinos dos nanocristais durante todo o experimento. Ao contrário do que se esperaria para nanocristais, a sinterização das partículas de $\mathrm{CeO}_{2}$ ocorreu em um alto patamar de temperatura. A temperatura de sinterização e a estabilidade dessas nanopartículas foram correlacionadas à formação de uma camada amorfa externa, proveniente da pirólise dos grupos oleatos ligados à superfície dos nanocristais. Adicionalmente, evidenciouse a importância na orientação cristalográfica entre os nanocristais em sinterização, sendo observada a reorientação das nanopartículas para o prosseguimento da sinterização.

\section{AGRADECIMENTOS}

Os autores agradecem os recursos financeiros fornecidos pela FAPESP (Procs. 09/50604-4 e 12/22974-4).

\section{REFERÊNCIAS}

[1] W. D. Kingery, H. K. Bowen, D. R. Uhlmann, Introduction to ceramics, Wiley (1976).

[2] R. M. German, Powder Metall. 56 (2013) 117.

[3] J. S. Reed, Principles of Ceramics Processing, John Wiley \& Sons (1995).

[4] J. R. Groza, Nanostructured Mater. 12 (1999) 987.

[5] C. Herring, J. Appl. Phys. 21 (1950) 301.

[6] H. Song, R. L. Coble, R. J. Brook, in Mater. Sci. Res., G. Kuczynski, A. Miller, G. Sargent, Eds., Springer US (1984), p 63.

[7] M. A. L. Cordeiro, P. A. Crozier, E. R. Leite, Nano Lett. 12 (2012) 5708.

[8] T. W. Hansen, J. B. Wagner, P. L. Hansen, S. Dahl, H. Topsoe, C. J. H. Jacobsen, Science 294 (2001) 1508.

[9] H. Poppa, J. Appl. Phys. 38 (1967) 3883.

[10] R. V. Latham, E. Braun, Vacuum 18 (1968) 410.

[11] M. O. Ruault, Philosophical Magazine 36 (1977) 835.

[12] J. Rankin, B. W. Sheldon, Mater. Sci. Eng. A - Struct. Mater. Prop. Microstruct. Process. 204 (1995) 48.

[13] J. Rankin, J. Am. Ceram. Soc. 82 (1999) 1560.

[14] W. J. Zhang, D. E. Miser, J. Nanopart. Res. 8 (2006) 1027.

[15] M. A. L. Codeiro, W. H. Weng, D. G. Stroppa, C. J. Kiely, E. R. Leite, Chem. Mater. 25 (2013) 2028.

[17] M. Ozawa, Scripta Mater. 50 (2004) 61.

[16] J. Luo, Current Opinion in Solid State \& Mater. Sci. 12 (2008) 81.

[18] Y. C. Zhou, M. N. Rahaman, Acta Mater. 45 (1997) 3635.

[19] Y. Kinemuchi, K. Watari, J. Eur. Ceram. Soc. 28 (2008) 2019.

[20] R. Di Monte, J. Kaspar, Topics in Catalysis 28 (2004) 47. 
[21] E. J. H. Lee, C. Ribeiro, E. Longo, E. R. Leite, J. Phys. Chem. B 109 (2005) 20842.

[22] R. Theissmann, M. Fendrich, R. Zinetullin, G. Guenther,
G. Schierning, D. E. Wolf, Phys. Rev. B 78 (2008) 205413.

[23] P. Grammatikopoulos, C. Cassidy, V. Singh, M. Sowwan, Sci. Reports 4 (2014).

(Rec. 15/04/2015, Ac. 29/05/2015) 